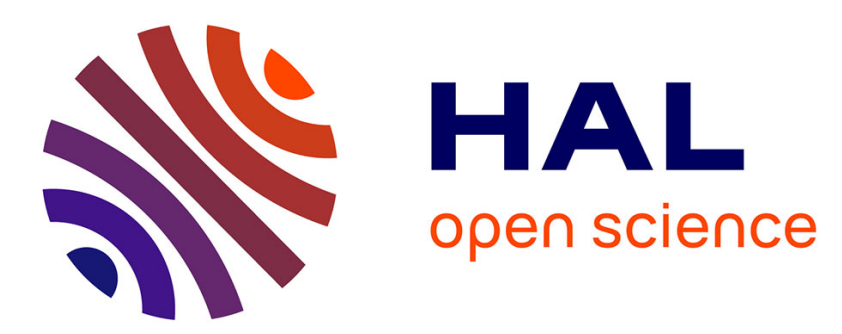

\title{
LMI-based design of a structured direct adaptive satellite attitude control with actuator rate feedback
}

Harmonie Leduc, Dimitri Peaucelle, Christelle Pittet-Mechin

\section{To cite this version:}

Harmonie Leduc, Dimitri Peaucelle, Christelle Pittet-Mechin. LMI-based design of a structured direct adaptive satellite attitude control with actuator rate feedback. 2015 IEEE 54th Annual Conference on Decision and Control (CDC 2015), Dec 2015, Osaka, Japan. 10.1109/CDC.2015.7402732 . hal01388191

\section{HAL Id: hal-01388191 \\ https://hal.science/hal-01388191}

Submitted on 28 Oct 2016

HAL is a multi-disciplinary open access archive for the deposit and dissemination of scientific research documents, whether they are published or not. The documents may come from teaching and research institutions in France or abroad, or from public or private research centers.
L'archive ouverte pluridisciplinaire HAL, est destinée au dépôt et à la diffusion de documents scientifiques de niveau recherche, publiés ou non, émanant des établissements d'enseignement et de recherche français ou étrangers, des laboratoires publics ou privés. 


\section{LMI-based design of a structured direct adaptive satellite attitude control with actuator rate feedback}

\author{
Harmonie Leduc \\ hleduc@laas.fr
}

\author{
Dimitri Peaucelle \\ peaucell@laas.fr
}

\author{
Christelle Pittet \\ christelle.pittet@cnes.fr
}

\begin{abstract}
Satellite attitude control of a microsatellite of the CNES Myriade series is considered. Reduction of saturations on the angular rate of reaction wheel actuators is addressed by means of control gain adaptation. The proposed adaptive control design has the following features: performances of the adaptive law are the same as those of a predefined linear time-invariant control when the state is close to the equilibrium; adaptation is structured allowing to include engineering considerations in the design; asymptotic stability is guaranteed based on Lyapunov arguments; fine tuning of adaptive gains is made by linear matrix inequality based convex optimization. The novelties compared to previous results are: to include magnetotorquer information in the adaptive scheme; to provide a simplified design procedure.
\end{abstract}

\section{Introduction}

The attitude control of satellites is a challenging issue that has attracted much attention of the scientific community (see for example $[23,3]$ ). Many of these contributions consider an idealized rigid body model with ideal actuators which allows to provide powerful global hybrid stabilization solutions such as in $[13,21]$. One can also cite sliding mode control solutions as for example [8] where additional flexible structure characteristics are included to the basic rigid body model, or [25] where actuator saturation is considered. Such non-linear approaches have the advantage of providing global stability characteristics essential when the satellite performs large atti- tude changes, but they can scarcely characterize the attitude control at fine pointing mode in which the satellite is most of the time.

A complementary approach is the design of a local linear control, for example using linear matrix inequality (LMI) based results as in [19, 2, 24]. Such approaches have the advantage to provide robust controllers with optimized performances that can designed for precise models including flexible modes and actuator dynamics. The main disadvantage of the obtained controllers is that they have high gain characteristics (for improved performance) that inevitably saturate the actuators as soon as the pointing errors are greater than a few degrees. To expand the neighborhood of the equilibrium point where the local linear control remains valid without saturating actuators, a solution adopted by CNES [16] is to switch to a less aggressive control when depointing exceeds some fixed threshold. In [1] a linear parameter-varying alternative is proposed that avoids the discontinuities due to switching. An other solution based on adaptive control is proposed in $[12,11]$ and was satisfactorily tested onboard the PICARD satellite [17]. The goal of the paper is to improve these last results and to justify theoretically some heuristic tunings done in [17].

At the difference of $[5,4,25]$, we adopt a direct adaptive control strategy that does not rely on online parameter estimation, but tunes directly the control gains based on the measurements. Such direct adaptive strategy follows the passivity-based results of $[6,9,7,14]$. Here we apply some of the most recent developments from [12] that have the following important features: the adaptive control design 
only requires feedback stabilizability of the plant (no closed-loop passivity assumption); the adaptive law can be structured which allows engineering considerations; the design of the adaptive law parameters is via linear matrix inequality (LMI) constrained optimization (that is convex and solvable efficiently in polynomial time).

The contributions of the paper are both in terms of improved attitude control strategy by adapting the magnetotorquer proportional gain, and in terms of reduction of the numerical burden of the LMI-based design.

Following the specifications [15] given by CNES (the french space agency that develops the Myriade series of micro satellites), and at the difference of many papers such as [25], the critical saturation issue that we consider is not in terms of saturation of the control torques, but the saturation of the reaction wheels angular rate. The actual control torque is related to the derivative of this angular rate and hence the satellite is uncontrollable by reaction wheels when saturation occurs. Our contribution is to include in the adaptive control strategy the information about the reaction wheels angular rate with the objective to avoid as much as possible the saturations.

The second contribution is to revisit the LMI-based design strategy. The new conditions we provide not only allow the design of the adaptive law parameters in one unique LMI step (two steps in [12]) but the adaptive control has proved asymptotic convergence to equilibrium points even in the presence of forgetting factor terms.

The paper is organized as follows. First we expose the satellite attitude control problem for the latest Myriade series satellite. Section III then provides the theoretical contributions, namely the LMI-based design methodology and the proofs of asymptotic stability of the direct adaptive control law. The fourth section demonstrates how the proposed methodology applies to the satellite example and illustrates the improvements made possible when adapting the magnetotorquer gain. Finally we draw some conclusions and perspectives for future work.

Notation. $I$ stands for the identity matrix. $A^{T}$ is the transpose of the matrix $A .\{A\}^{\mathcal{S}}$ stands for the symmetric matrix $\{A\}^{\mathcal{S}}=A+A^{T} . A(\preceq) \prec B$ is the matrix inequality stating that $A-B$ is negative (semidefinite. $\operatorname{diag}\left(F_{1}, \cdots, F_{\bar{k}}\right)$ stands for a bloc-diagonal matrix whose diagonal blocs are the $F_{1}, \ldots F_{k}, \ldots F_{\bar{k}}$ matrices.

\section{Taranis microsatellite atti- tude control}

In 2016-2017, CNES schedules to send to orbit an other microsatellite from the Myriade series, Taranis, dedicated to the study of thunderstorms. The satellite will weight from 150 to $200 \mathrm{~kg}$ and its architecture is very close to that of Demeter [15] satellites launched in 2004. It is more complex to control than the Picard satellite launched in 2010 since it has four appendices (as for Demeter) that bring low frequency flexible modes to the dynamics. The current study is assuming the dynamics of the three axes are decoupled and we consider the linearized $\mathrm{x}$-axis model with flexible modes:

$$
\theta=G_{T a r}(s) T=\frac{0.449 s^{2}+0.0038 s+1}{2.3169 s^{4}+0.1425 s^{3}+37.49 s^{2}} T .
$$

The actuation torque $T$ is realized by reaction wheels modeled as

$$
\begin{aligned}
T & =G_{r}(s) \omega_{r s}=\frac{(1.214 s+0.7625) J_{r} s}{s^{2}+2.4 s+0.7625} \omega_{r s}, \\
\omega_{r} & =\frac{1}{J_{r} s} u_{f}
\end{aligned}
$$

where $\omega_{r}$ is the reaction wheel angular rate and $J_{r}$ is the inertia of the reaction wheel. In practice the reaction wheel is saturated in terms of angular rate: $\omega_{r s}=\operatorname{sat}_{\bar{\omega}_{r}}\left(\omega_{r}\right)$ where $\pm \bar{\omega}_{r}= \pm 293 \mathrm{rad} / \mathrm{s}$ are the limits on the angular rate. Due to the derivative type term in $G_{r}(s)$, as soon as the reaction wheel rate saturates, the actuation torque $T$ is zero: the satellite is non actuated. It is a situation to be avoided.

The classical control law structure for the Myriade series is composed of an estimator of the satellite angular rate $\omega_{e}=G_{e}(s) \theta$, a proportional/derivative baseline control $u_{c}=F_{\theta} \theta+F_{\omega} \omega_{e}$ and some stabilizing fourth order filter $u_{f}=G_{f}(s) u$. The same filter is considered as in [16]. This classical structure suffers 


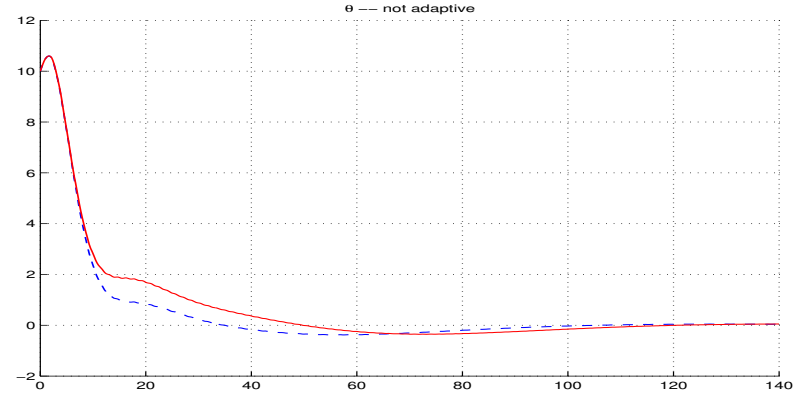

Figure 1: Attitude angle. Dashed: classical control architecture; Solid: with the magnetotorquer.

from the fact that the reaction wheel rate is a non asymptotically stable state. To stabilize it without introducing much difference with the existing control architecture, we also consider in this paper case the action of a magnetotorquer, whose transfer function is a proportional-integral controller:

$T=\left(G_{r}(s)+J_{r} G_{m g t}(s)\right) \omega_{r s}, \quad G_{m g t}(s)=0.01+\frac{6 e^{-5}}{s}$.

To illustrate the modification brought by the magnetotorquer the time histories of the satellite angle, angular rate and the reaction wheel angular rate are plotted respectively in Figures 1, 2 and 3. The initial conditions $\theta(0)=10 \mathrm{deg}, \omega(0)=0.01 \mathrm{rad} / \mathrm{s}$ and $\omega_{r}(0)=0 \mathrm{rad} / \mathrm{s}$ are such that the reaction wheel saturates. The angular rate shows some rapid oscillations due to the flexible mode. The proportional-integral controller improves the dynamics of the response and reduces slightly the time of saturation, at the expense of a slower stabilization of the reaction wheel speed (the magnetotorquer uses magnetic field data). Our goal is to reduce it further, without modifying the characteristics of the closed-loop at fine foisting. For that purpose we propose in the following a rigorous LMI-based methodology to design an adaptive version of this controller.

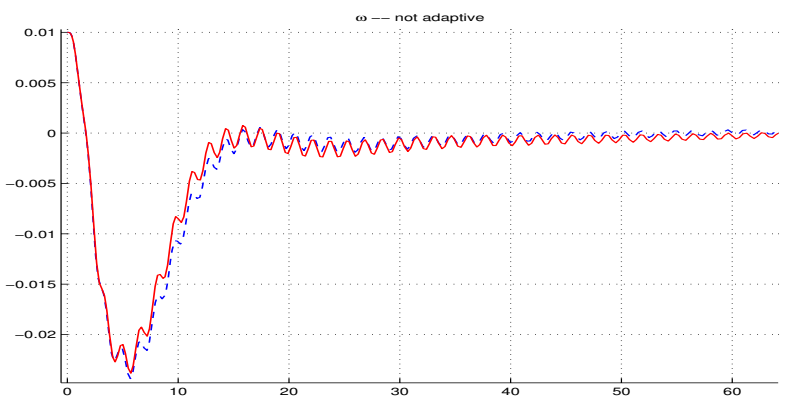

Figure 2: Attitude angular rate. Dashed: classical control architecture; Solid: with the magnetotorquer.

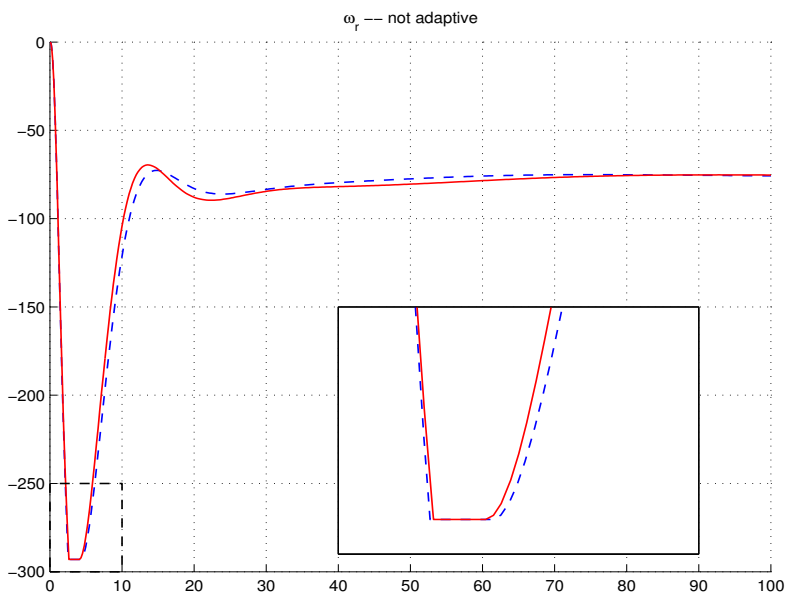

Figure 3: Actuator angular rate. Dashed: classical control architecture; Solid: with the magnetotorquer. 


\section{New LMI-based adaptive control design}

Let the following linear state-space model for the plant

$$
\dot{x}=A x+B u, \quad y=C x
$$

and assume some existing structured stabilizing static output feedback control written in the following format

$$
u=\sum_{k=1}^{\bar{k}} L_{k} F_{k} R_{k} y
$$

where $L_{k}$ and $R_{k}$ are full rank matrices composed of zero or unity elements, defining the structure of the controller, and where $F_{k}$ are full-block independent gains. For building compact formulas we denote $L=$ $\left[\begin{array}{lll}L_{1} & \cdots & L_{\bar{k}}\end{array}\right], R^{T}=\left[\begin{array}{lll}R_{1}^{T} & \cdots & R_{\bar{k}}^{T}\end{array}\right]$ and $F=$ $\operatorname{diag}\left(F_{1}, \ldots, F_{\bar{k}}\right)$ the block-diagonal matrix composed of all the gains. With these notations one has $u=$ LFRy

For the attitude control example, the output vector is $y^{T}=\left(\begin{array}{lll}\omega_{r} & \theta & \omega_{e}\end{array}\right)$ and the control input is $u^{T}=$ $\left(\begin{array}{ll}u_{r} & u_{c}\end{array}\right)$. The considered controller structure is such that

$L F R=\left[\begin{array}{l|l|l}1 & 0 & 0 \\ 0 & 1 & 1\end{array}\right]\left[\begin{array}{ccc}F_{r} & 0 & 0 \\ 0 & F_{\theta} & 0 \\ 0 & 0 & F_{\omega}\end{array}\right]\left[\begin{array}{lll}1 & 0 & 0 \\ \hline 0 & 1 & 0 \\ \hline 0 & 0 & 1\end{array}\right]$

The proposed structured adaptive control amounts to replacing the static gains $F_{k}$ by dynamically adapted ones such that $u(t)=L K(t) R y(t)$ with $K(t)=\operatorname{diag}\left(K_{1}(t), \ldots, K_{\bar{k}}(t)\right)$ and each individual gain $K_{k}(t)$ is solution to non-linear differential equations

$$
\begin{aligned}
& \dot{K}_{k}(t)=\operatorname{Proj}_{D_{k}}\left(K_{k}(t)-F_{k}, W_{k}(t)\right) \\
& W_{k}(t)=\gamma_{k}\left(-G_{k} y(t)\left(R_{k} y(t)\right)^{T}-\sigma_{k}\left(K_{k}(t)-F_{k}\right)\right) .
\end{aligned}
$$

In these equations the $\operatorname{Proj}_{D_{k}}$ operator is parameterized by a matrix $D_{k}$ that defines an ellipsoidal-like set defined by the following inequality:

$$
\left(K_{k}-F_{k}\right) \in \mathcal{E}_{k} \Leftrightarrow \operatorname{Tr}\left(\left(K_{k}-F_{k}\right)^{T} D_{k}\left(K_{k}-F_{k}\right)\right) \leq 1
$$

The operator $\operatorname{Proj}_{D_{k}}$ has a similar definition as in [20]. It outputs the value $W_{k}$ when the gain $K_{k}-F_{k}$ is in the interior of the set and enforces the derivative of $K_{k}$ to be such that the gain is pushed to the interior of the set when it is at the border:

$$
\operatorname{Proj}_{D_{k}}\left(K_{k}-F_{k}, W_{k}\right)=W_{k}-H_{k}
$$

where $H_{k}$ is such that

$$
\begin{aligned}
& H_{k}=0 \text { if }\left(K_{k}-F_{k}\right) \in \mathcal{E}_{k} \\
& \text { else s.t. }\left\{\begin{array}{l}
\operatorname{Tr}\left(\left(W_{k}-H_{k}\right)^{T} D_{k}\left(K_{k}-F_{k}\right)\right) \leq 0 \\
\operatorname{Tr}\left(\left(K_{k}-F_{k}\right)^{T} H_{k}\right) \geq 0
\end{array}\right.
\end{aligned}
$$

The first of the two inequalities in (8) ensures that the derivative of $\operatorname{Tr}\left(\left(K_{k}-F_{k}\right)^{T} D_{k}\left(K_{k}-F_{k}\right)\right)$ is negative when $\operatorname{Tr}\left(\left(K_{k}-F_{k}\right)^{T} D_{k}\left(K_{k}-F_{k}\right)\right)=1$. Hence the gains cannot exit the set $\mathcal{E}_{k}$. The second imposes $H_{k}$ to be oriented towards the exterior of the set (no need so subtract any component if $W_{k}$ already pushes the gain to the interior of the set).

The operator guarantees that whatever values of $W_{k}$, the gains $K_{k}$ remain in a bounded neighborhood of $F_{k}$ parameterized by $D_{k}$. In the case of scalar gains $K_{k}$ the set $\mathcal{E}_{k}$ is an interval and the $\operatorname{Proj}_{D_{k}}$ operator can be implemented as a saturated integrator.

The adaptation in (6) is driven by two terms. The first term $G_{k} y(t)\left(R_{k} y(t)\right)^{T}$ drives the adaptation while the second term $-\sigma_{k}\left(K_{k}(t)-F_{k}\right)$ is a forgetting factor that brings the gain back to the value $F_{k}$ as soon as the system is at the zero equilibrium. The adaptation law hence has the property that the control is exactly the same as the baseline control $F$ when the errors are small (at least very close to it). Any property of the control $F$ computed on the linear model remains valid for the adaptive control, at least for small deviations from the equilibrium point where the linear model is valid.

The design problem for the adaptive law (6) is to choose appropriately the matrices $D_{k}, G_{k}$ and the scalars $\sigma_{k}, \gamma_{k}$. The following theorem answers this question.

Theorem 3.1 If LFR is an asymptotically stabilizing gain for the plant (4), then there exist $P \succ$ $0, \epsilon>0, G=\left[\begin{array}{lll}G_{1}^{T} & \cdots & G_{\bar{k}}\end{array}\right]^{T}$ and $D=$ 
$\operatorname{diag}\left(D_{1}, \ldots, D_{\bar{k}}\right)$ solution to

$$
\left[\begin{array}{cc}
\{P(A+B L F R C)\}^{\mathcal{S}}+\epsilon I+2 C^{T} R^{T} R C & P B L-C^{T} G^{T} \\
L^{T} B^{T} P-G C & -2 D
\end{array}\right] \underset{2 y}{{ }^{T}} R^{T}\left(I-(K-F)^{T} D(K-F)\right) R y \leq-\epsilon x^{T} x .
$$

Moreover, the solution is such that the adaptive control (6) stabilizes the plant whatever positive values of $\sigma_{k}, \gamma_{k}$.

Proof: The first part of the proof is to assess that if the stability property holds for the static gain $F$, then there indeed exist $\epsilon, D_{k}$ and $G_{k}$ parameters solution to the LMI.

Let $\hat{V}(x)=x^{T} \hat{P} x, \hat{P} \succ 0$ be a quadratic Lyapunov function which proves the asymptotic stability of $\dot{x}=$ $(A+B L F R C) x$, i.e. $\{\hat{P}(A+B L F R C)\}^{\mathcal{S}} \prec 0$. Ву а small perturbation argument, and whatever a priori choice of $\hat{G}$, there exist small positive $\breve{\epsilon}>0, \hat{\epsilon}>0$ and $\tilde{\epsilon}>0$ such that

$$
\begin{aligned}
\{\hat{P}(A+B L F R C)\}^{S} \preceq & -\breve{\epsilon} I-\hat{\epsilon} C^{T} R^{T} R C \\
& -\tilde{\epsilon}\left(\hat{P} B L-C^{T} \hat{G}^{T}\right)\left(L^{T} B^{T} \hat{P}-\right.
\end{aligned}
$$

Multiply this inequality by $\kappa=2 / \hat{\epsilon}$ and take $\epsilon=\kappa \breve{\epsilon}$, $P=\kappa \hat{P}, G=\kappa \hat{G}, D=(\hat{\epsilon} / \tilde{\epsilon}) I$, the inequality also reads as

$$
\begin{aligned}
\{P(A+B L F R C)\}^{S} \preceq-\epsilon I-2 C^{T} R^{T} R C \\
-\left(P B L-C^{T} G^{T}\right)(2 D)^{-1}\left(L^{T} B^{T} P\right.
\end{aligned}
$$

By a Schur complement argument, this inequality is equivalent to the LMI (9).

Now let us prove the stability of the closed-loop with adaptive control. Pre an post-multiply (9) by $\left(x^{T} x^{T} C^{T} R^{T}(K-F)^{T}\right)$ and its transpose respectively, to get for $y=C x$ :

$$
\begin{aligned}
2 x^{T} P A x+2 & x^{T} P B L F R y+\epsilon x^{T} x+2 y^{T} R^{T} R y \\
+ & 2 x^{T}\left(P B L-C^{T} G^{T}\right)(K-F) R y \leq 0 . \\
& -2 y^{T} R^{T}(K-F)^{T} D(K-F) R y
\end{aligned}
$$

Along the adaptive control closed-loop system trajectories $\dot{x}=(A+B L K R C) x$, this inequality also reads as

$$
\begin{aligned}
& 2 x^{T} P \dot{x}-x^{T} P B L(K-F) R y+2 y^{T} R^{T} R y \\
& +2 x^{T}\left(P B L-C^{T} G^{T}\right)(K-F) R y \leq-\epsilon x^{T} x \\
& -2 y^{T} R^{T}(K-F)^{T} D(K-F) R y
\end{aligned}
$$

Due to the block-diagonal nature of the matrices $K$, $F$ and $D$, the matrix $\left.I-(K-F)^{T} D(K-F)\right)$ is block diagonal with $\left.I-\left(K_{k}-F_{k}\right)^{T} D_{k}\left(K_{k}-F_{k}\right)\right)$ elements on its diagonal. Moreover recall that for a positive definite matrix $M, \operatorname{Tr}(M) \leq 1$ implies $I-M \succeq 0$. Because of this one has:

$$
2 x^{T} P \dot{x}-2 y^{T} G^{T}(K-F) R y \leq-\epsilon x^{T} x .
$$

We shall now exploit (10) to prove stability of the adaptive control closed-loop. For that purpose consider the following Lyapunov function:

$$
V(x, K)=x^{T} P x+\operatorname{Tr}\left((K-F)^{T} \Gamma^{-1}(K-F)\right)
$$

where $\Gamma=\operatorname{diag}\left(\gamma_{1} I_{m_{1}}, \cdots, \gamma_{\bar{k}} I_{m_{\bar{k}}}\right)$ and $m_{k}$ is the number of columns of $L_{k}$. The derivative of this Lyafump function along the trajectories of the adaptive closed-loop system reads as

$$
\begin{aligned}
\dot{V}(x, K)= & 2 x^{T} P \dot{x}+2 \operatorname{Tr}\left((K-F)^{T} \Gamma^{-1} \dot{K}\right) \\
= & 2 x^{\prime} P \dot{x}-2 \operatorname{Tr}\left((K-F)^{T}(G y)(R y)^{T}\right) \\
& -2 \operatorname{Tr}\left((K-F)^{T} \sigma(K-F)\right)-2 \operatorname{Tr}\left((K-F)^{T} \Gamma^{-1} H\right)
\end{aligned}
$$
ing the derivative of the adaptive add-on by its formula, while taking $\sigma=\operatorname{diag}\left(\sigma_{1} I_{m_{1}}, \ldots, \sigma_{\bar{k}} I_{m_{\bar{k}}}\right)$ and $H=\operatorname{diag}\left(H_{1}, \ldots, H_{\bar{k}}\right)$. Using the properties of the trace operator, the fact that matrices are block diagonal and the definition of the $H_{k}$ matrices one gets:

$$
\operatorname{Tr}\left((K-F)^{T} \Gamma^{-1} H\right)=\sum_{k=1}^{\bar{k}} \gamma_{k}^{-1} \operatorname{Tr}\left(\left(K_{k}-F_{k}\right)^{T} H_{k}\right) \geq 0 .
$$

Moreover, thanks to the trace operator properties on has

$$
\begin{aligned}
\operatorname{Tr}\left((K-F)^{T}(G y)(R y)^{T}\right) & =\operatorname{Tr}\left((R y)(G y)^{T}(K-F)\right) \\
& =\operatorname{Tr}\left((G y)^{T}(K-F)(R y)\right) \\
& =y^{T} G^{T}(K-F) R y
\end{aligned}
$$

Hence using (10), the derivative of the Lyapunov function is negative: mesecond row of this formula is obtained by replac- 
According to the Lyapunov theory the non-linear adaptive closed-loop system is asymptotically stable and the states $x$ and $K_{k}$ converge respectively to zero and $F_{k}$.

Remark 1: The $D_{k}$ matrices parametrize the sets in which the adaptive gains lie. The "larger" $D_{k}$ is, then the smaller is the set $\mathcal{E}_{k}$. This means that if we aim at having large sets in which the adaption performs, one has to look for the "smallest" possible $D_{k}$. In practice we suggest to minimize

$$
f_{w}(D)=\sum_{k=1}^{\bar{k}} w_{k} \operatorname{Tr}\left(D_{k}\right)
$$

where the weights $w_{k}$ are chosen as a tradeoff between the different gains and the trace operator is chosen such that the "size" is defined by a linear function. Minimizing $f_{w}(D)$ under the LMI constrains (10) is a convex optimization problem that can be easily coded in Matlab using the YALMIP parser [10] and solved efficiently using semi-definite programming tools such as [22].

Remark 2: The condition (10) being linear in $G$, it is possible to perform the convex LMI optimization with any additional linear constraints on the coefficients of $G$. This is used in the following for the satellite example in order to force the directions in which adaptation is done, based on engineering considerations.

Remark 3: Theorem 3.1 is an improved version of results given in [12]. The improvements have the following characteristics

- The LMI-based design is done in one step (two steps in [12]) thus reducing the computation burden by a factor two.

- The adaptive gains are guaranteed to converge asymptotically to the nominal values $F_{k}$, while in [12] only convergence to a neighborhood of these is guaranteed. The drawback is that the sets in which the gains evolve may be smaller.

- In [12] boundedness of the adaptive gains is guaranteed by a penalty barrier function in the adaptation equation. Such barrier function has proved complex to implement, even in the case of scalar gains (see [11]). The projection operator appears to be more appropriate for implementation.

\section{Design of the Taranis adap- tive attitude control}

By default the adaptive law for the satellite control with three scalar gains is driven by the following equations

$$
\begin{aligned}
& W_{\theta}(t)=\gamma_{\theta}\left(-G_{\theta} y(t) \theta(t)-\sigma_{\theta}\left(K_{\theta}(t)-F_{\theta}\right)\right) \\
& W_{\omega}(t)=\gamma_{\omega}\left(-G_{\omega} y(t) \omega_{e}(t)-\sigma_{\omega}\left(K_{\omega}(t)-F_{\omega}\right)\right) \\
& W_{m g t}(t)=\gamma_{m g t}\left(-G_{m g t} y(t) h_{r}(t)-\sigma_{m g t}\left(K_{m g t}(t)-F_{m g t}\right)\right) \\
& h_{r}(t)==J_{r} \omega_{r}(t) .
\end{aligned}
$$

and the projection operator guarantees the gains to be in intervals centered at the nominal values and of length proportional to the inverse of the square root of the $D$ matrices:

$$
\begin{aligned}
& K_{\theta} \in\left[F_{\theta}-D_{\theta}^{-1 / 2}, F_{\theta}+D_{\theta}^{-1 / 2}\right], \\
& K_{\omega} \in\left[F_{\omega}-D_{\omega}^{-1 / 2}, F_{\omega}+D_{\omega}^{-1 / 2}\right], \\
& K_{m g t} \in\left[F_{m g t}-D_{m g t}^{-1 / 2}, F_{m g t}+D_{m g t}^{-1 / 2}\right] .
\end{aligned}
$$

\subsection{Design without actuator rate feedback}

To analyse the usefulness of the magnetotorquer introduced in section 2 for the adaptive control problem, we first consider the case when only position and rate are adapted. $F_{m g t}=-0.01$ is constant and $G_{m g t}=0$. The LMI problem is then solved with the following constraints on the other matrices:

$$
\begin{array}{ll}
G_{\theta}=\left[\begin{array}{ccc}
g_{\theta} & 0 & 0
\end{array}\right], & g_{\theta} \geq 1, \\
G_{\omega}=\left[\begin{array}{lll}
0 & g_{\omega} & 0
\end{array}\right], & g_{\omega} \leq-10 g_{\theta} .
\end{array}
$$

This choice is done such that: when the depointing is large $\left(\theta^{2}\right.$ is large), the adaptation will push $K_{\theta}$ to smaller values, thus reducing the actuation effort; when the satellite rotation rate is large ( $\omega_{e}^{2}$ is large), the adaptation will push $K_{\omega}$ to larger values, thus regulating strongly the angular rate to zero. Both 
these effects tend to slow down the convergence of the attitude with the expected benefit of avoiding actuator rate saturation. The factor 10 between the two gains is chosen heuristically to avoid numerical issues. It has no influence on the adaptive control itself since the adaptation equations (6) are finally multiplied by a positive scalar $\gamma_{k}$ that drives the adaptation speed of each gain independently.

Weights chosen as $w_{\theta}=10, w_{\omega}=1$ in order to push for larger variations of $K_{\theta}$ compared to those on $K_{\omega}$. The LMI optimization problem is solved with a computation time of about 1 second and gives:

$$
\begin{gathered}
g_{\theta}=2.52, \quad D_{\theta}^{-1 / 2}=0.0986, \\
g_{\omega}=-25.19, \quad D_{\omega}^{-1 / 2}=0.0517 .
\end{gathered}
$$

The other parameters are chosen accordingly to the methodology exposed in [12]. Their values are

$$
\gamma_{\theta}=0.569, \quad \sigma_{\theta}=0.049, \quad \gamma_{\omega}=0.569, \quad \sigma_{\omega}=0.037 .
$$

Simulation results with this adaptive control are plotted with dash lines in Figures 4, 5, 6, 7 and 8.

\subsection{Design with actuator rate feed- back}

In [17] a modification of the adaptive law is proposed (and successfully tested onboard the PICARD satellite). This modification is heuristic and comes from the intuition that there is no reason for slowing down the dynamics when the reactions wheel angular rate is far from saturation. The proposed modification is on the adaptive rule for $K_{\theta}$ and reads as

$$
W_{\theta}(t)=\gamma_{\theta}\left(-g_{\theta} \theta^{2}(t)+g_{r} \omega_{r}^{2}-\sigma_{\theta}\left(K_{\theta}(t)-F_{\theta}\right)\right),
$$

Rather than testing this heuristic solution, we suggest a new one that can be validated with the LMI design and that assumes that the proportional gain $K_{p}$ of the proportional-integral controller of the magnetotorquer can be adapted as the gains $K_{\theta}$ and $K_{\omega}$ are.

The design is lead with the following constraints

$$
\begin{array}{ll}
G_{\theta}=\left[\begin{array}{ccc}
g_{\theta} & 0 & 0
\end{array}\right], & g_{\theta} \geq 0, \\
G_{\omega}=\left[\begin{array}{lll}
0 & g_{\omega} & 0
\end{array}\right], & g_{\omega} \leq 0, \\
G_{m g t}=\left[\begin{array}{lll}
0 & 0 & g_{m g t}
\end{array}\right], & g_{m g t} \leq 0
\end{array}
$$

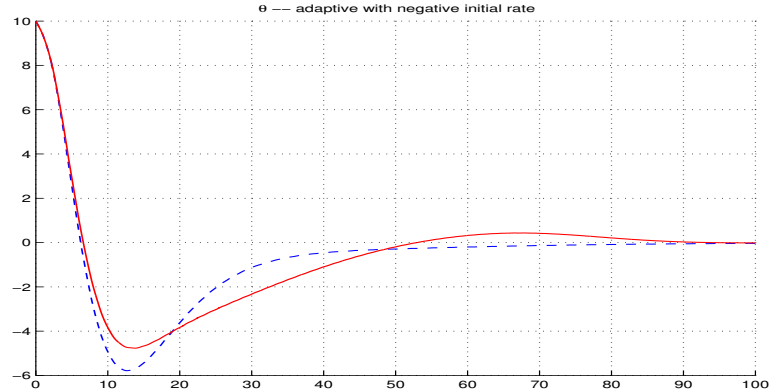

Figure 4: Attitude angle. Dashed: without mgt proportional gain adaptation; Solid: with mgt proportional gain adaptation.

Weights are chosen to fit the previous ones $w_{\theta}=10, w_{\omega}=1$ and with $w_{m g t}=10^{-4}$ that need not to be large since $K_{m g t}$ will vary around small values $\left(F_{m g t}=-10^{-2}\right)$. The LMI optimization problem is solved with a computation time of about 1 second and gives:

$$
\begin{gathered}
g_{\theta}=103.64, \quad D_{\theta}^{-1 / 2}=0.0411 \\
g_{\omega}=-1039.39, \quad D_{\omega}^{-1 / 2}=0.0179 \\
g_{m g t}=-163.85, \quad D_{m g t}^{-1 / 2}=0.0020
\end{gathered}
$$

The other parameters are chosen accordingly to the methodology exposed in [12]. Their values are

$$
\begin{gathered}
\gamma_{\theta}=0.014, \quad \sigma_{\theta}=4.801, \quad \gamma_{\omega}=0.014, \quad \sigma_{\omega}=4.424, \\
\gamma_{m g t}=0.007, \quad \sigma_{m g t}=613.137 .
\end{gathered}
$$

Simulation results with this adaptive control are plotted with solid lines in Figures 4, 5, 6, 7 and 8.

\subsection{Simulation results}

Time responses of the attitude angle with the same initial conditions as those used in Section II are plotted in Figure 4. The comparison with Figure 1 shows that the adaptive controllers allow a faster convergence.

Time responses of the actuator angular rate are plotted in Figure 5. The comparison with Figure 3 shows that the adaptive controllers allow a reduced 


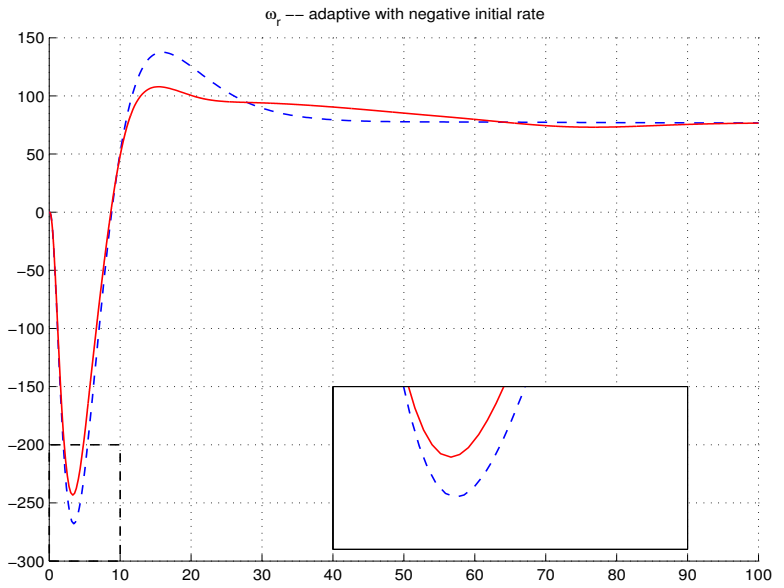

Figure 5: Actuator angular rate. Dashed: without mgt proportional gain adaptation; Solid: with mgt proportional gain adaptation.

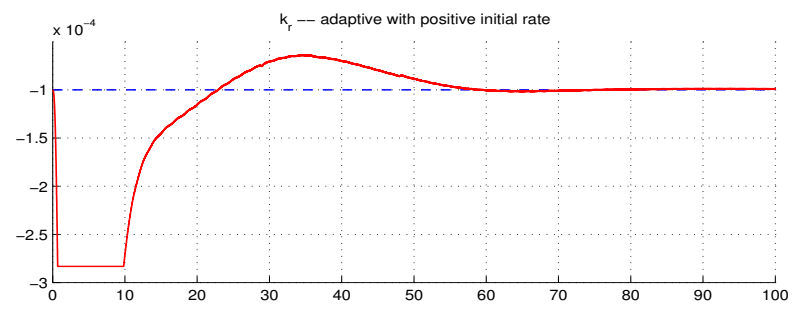

Figure 6: Adaptive gain $K_{m g t}$. Dashed: without mgt proportional gain adaptation; Solid: with mgt proportional gain adaptation.

saturation time. The adaptation based on the reaction wheel momentum further reduces the saturation time.

Time histories of the adaptive gain $K_{m g t}$ are plotted in Figure 6. The dotted curve is constant since it corresponds to the case when no adaptation is done for this gain. In the adapted case the variations are non negligible compared to the nominal value.

Time histories of the gains $K_{\theta}$ and $K_{\omega}$ are plotted in Figures 7 and 8 respectively. In the case of adaptation involving the actuator rate the bounds in which the gains evolve are tighter. This is coherent with the smaller values of $D_{\theta}^{-1 / 2}$ and $D_{\omega}^{-1 / 2}$. The new

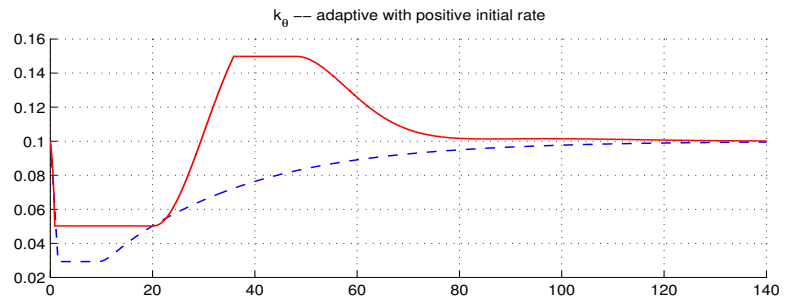

Figure 7: Adaptive gain $K_{\theta}$. Dashed: without mgt proportional gain adaptation; Solid: with mgt proportional gain adaptation.

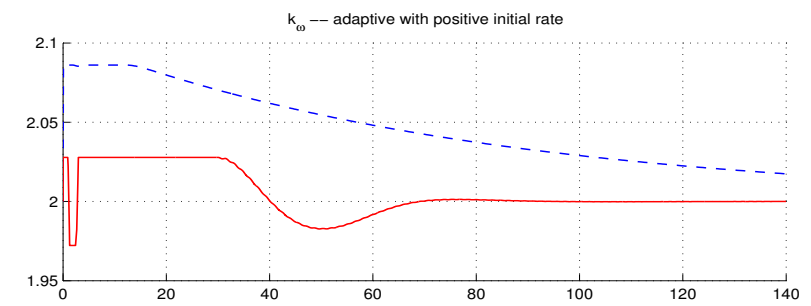

Figure 8: Adaptive gain $K_{\omega}$. Dashed: without mgt proportional gain adaptation; Solid: with mgt proportional gain adaptation.

adaptive strategy achieves better performances without having too large valuations of the gains around their nominal values.

An important feature is that the gains do converge back to their nominal values as soon as the satellite is close to the equilibrium. The convergence rate is driven by the $\sigma_{\bullet}$ values (see [12] for the explanation on how these should be chosen based on thresholds between large and small distances to equilibrium). An other feature is that the gains evolve rather smoothly. This is achieved by tuning the $\gamma$ • values knowing the range of evolution of the different signals, in order to have reasonable, implementable, values for the derivatives $\dot{K}_{k}$.

\section{CONCLUSIONS AND FU- TURE WORKS}

We have explored in this paper the opportunity to adapt control gains based on all available information 
from the plant: attitude angle and rate, plus actuator rate. Results show that such adaptive control can be designed based on any existing linear-time invariant control, that performances of this baseline control are recovered when the state is close to equilibrium, and that the design can be lead using up-to-date convex optimization techniques. In terms of the application, the adaptive control improves slightly the criterion that we have considered (faster time response and reduction of the actuator rate saturation). This feature needs to be validated on a 3 axis non-linear model of the satellite which is left for future work. The advantage of the linear-matrix inequality based design is that, except for possible numerical issues, it should be applicable to full 3 axis models.

\section{References}

[1] J.-M. Biannic, C. Roos, and C. Pittet. LPV analysis of switched controllers for attitude control systems. Journal of Guidance, Control and Dynamics, 34(5):1561-1566, 2011.

[2] C. Charbonnel. $H_{\infty}$ and LMI attitude control design: towards performances and robustness enhancement. Acta Astronautica, 54:307-314, 2004

[3] N.A. Chaturvedi, A.K. Sanyal, and N.H. McClamroch. Rigid-body attitude control. IEEE Control Systems Magazine, 31(3):30-51, 2011.

[4] B.T. Costic, D.M. Dawson, M.S. de Queiroz, and V. Kapila. A quaternion-based adaptive attitude tracking controller without velocity measurements. Journal of Guidance, Control and Dynamics, 24(6):1214-1222, 2001.

[5] O. Egeland and J.-M. Godhavn. Passivity based adaptive attitude control of a rigid spacecraft. IEEE Transactions on Automatic Control, 39(4):842-846, 1994.

[6] A.L. Fradkov. Adaptive stabilization of a linear dynamic plant. Autom. Remote Contr., 35(12):1960-1966, 1974.
[7] A.L. Fradkov, I.V. Miroshnik, and V.O. Nikiforov. Nonlinear and Adaptive Control of Complex Systems. Kluwer Academic Publishers, Dordrekht, 1999.

[8] Qinglei Hu. Sliding mode attitude control with $L_{2}$-gain performance and vibration reduction of flexible spacecraft with actuator dynamics. Acta Astronautica, 67:572-583, 2010.

[9] H. Kaufman, I. Barkana, and K. Sobel. Direct adaptive control algorithms. Springer, New York, 1998. Second Edition.

[10] J. Löfberg. YALMIP : A toolbox for modeling and optimization in MATLAB, 2001.

[11] A.R. Luzi. Commande variante dans le temps pour le contrôle d'attitude de satellites. $\mathrm{PhD}$ thesis, Université de Toulouse, February 2014.

[12] A.R. Luzi, D. Peaucelle, J.-M. Biannic, C. Pittet, and J. Mignot. Structured adaptive attitude control of a satellite. Int. J. of Adaptive Control and Signal Processing, 28(7-8):664-685, 2014.

[13] C.G. Mayhew, R.G. Sanfelice, and A.R. Teel. Quaternion-based hybrid control for robust global attitude tracking. IEEE Transactions on Automatic Control, pages 2555 - 2566, 2011.

[14] D. Peaucelle and A.L. Fradkov. Robust adaptive $L_{2}$-gain control of polytopic MIMO LTI systems - LMI results. Systems \& Control Letters, 57(11):881-887, 2008.

[15] C. Pittet and D. Arzelier. DEMETER: a benchmark for robust analysis and control of the attitude of flexible microsatellites. In IFAC Symposium on Robust Control Design, Toulouse, France, 2006.

[16] C. Pittet and C. Fallet. Gyroless attitude control of a flexible microsatellite. In Dynamics and control of systems and structures in space, pages 65-71, Cambridge, UK, 2002.

[17] C. Pittet, A.R. Luzi, D. Peaucelle, J.-M. Biannic, and J. Mignot. In flight results of adaptive 
attitude control law for a microsatellite. CEAS Space Journal, December 2014. Journal version of [18].

[18] C. Pittet, A.R. Luzi, D. Peaucelle, J.-M. Biannic, and J. Mignot. In flight results of adaptive attitude control law for a microsatellite. In ESA Conference on Guidance, Navigation and Control Systems, Porto, June 2014.

[19] C. Pittet, J. Mignot, and C. Fallet. LMI based multi-objective $H_{\infty}$ control of flexible microsatellites. In IEEE Conference on Decision and Control, Sydney, Australia, 1999.

[20] J.B. Pomet and L. Praly. Adaptive nonlinear regulation: Equation error from the lyapunov function. In IEEE Conf. Decision and Control, 1989.

[21] R. Schlanbusch, A. Loria, and P.J. Nicklasson. On the stability and stabilization of quartenion equilibria of rigid bodies. Automatica, 48(12):3135-3141, 2012.

[22] T.C. Toh, M.J. Todd, and R.H. Tutuncu. SDPT3 - a MATLAB software package for semidefinite programming. Optimization Methods and Software, 11:545-581, 1999.

[23] J.T.Y Wen and K. Kreutz-Delgado. The attitude control problem. IEEE Transactions on Automatic Control, 36(10):1148-1162, 1991.

[24] B. Wu, X. Cao, and Z. Li. Multi-objective output-feedback control for microsatellite attitude control: an LMI approach. Acta Astronautica, 64:1021-1031, 2009.

[25] Z. Zhu, Y. Xia, and M. Fu. Adaptive sliding mode control for attitude stabilization with actuator saturation. IEEE Transactions on Industrial Electronics, 58:4898-4907, 2011. 